\title{
Interactions between genetic variants of folate metabolism genes and lifestyle affect plasma homocysteine concentrations in the Boston Puerto Rican population
}

\author{
Tao Huang ${ }^{1,2,3}$, Katherine L Tucker ${ }^{4}$, Yu-Chi Lee ${ }^{2}$, Jimmy W Crott $^{2}$, Laurence D Parnell ${ }^{2}$, \\ Jian Shen ${ }^{2}$, Caren E Smith ${ }^{2}$, Jose M Ordovas ${ }^{2}$, Duo Li ${ }^{1,3, *}$ and Chao-Qiang Lai ${ }^{2, *}$ \\ 'Department of Food Science and Nutrition, Zhejiang University, Hangzhou, People's Republic of China: ${ }^{2} J e a n$ \\ Mayer-USDA Human Nutrition Research Center on Aging at Tufts University, Boston, 711 Washington Street, \\ Boston, MA 02111 , USA: ${ }^{3}$ APCNS Centre of Nutrition and Food Safety, Hangzhou, People's Republic of China: \\ ${ }^{4}$ Department of Health Sciences, Northeastern University, Boston, MA, USA
}

Submitted 14 September 2010: Accepted 20 December 2010: First published online 22 February 2011

\begin{abstract}
Objective: To investigate genetic and lifestyle factors and their interactions on plasma homocysteine (Hcy) concentrations in the Boston Puerto Rican population. Design: Cross-sectional study. Plasma concentrations of Hcy, folate, vitamin $\mathrm{B}_{12}$ and pyridoxal phosphate were measured, and genetic polymorphisms were determined. Data on lifestyle factors were collected in interviews.

Setting: A population survey of health and nutritional measures.

Subjects: A total of 994 Puerto Rican men and women residing in the Boston metropolitan area.

Results: Smoking status was positively associated with plasma Hcy. Genetic polymorphisms MTHFR 677C $\rightarrow$ T, FOLH1 1561C $\rightarrow$ T, FOLH1 rs647370 and PCFT 928A $\rightarrow$ $\mathrm{G}$ interacted significantly with smoking for Hcy. MTHFR $1298 \mathrm{~A} \rightarrow \mathrm{C}(P=0 \cdot 040)$ and $P C F T$ 928A $\rightarrow \mathrm{G}(P=0 \cdot 002)$ displayed significant interactions with alcohol intake in determining plasma Hcy. Subjects with PCFT 928GG genotype had significantly higher plasma Hcy concentrations compared with carriers of the $A$ allele $(A A+A G$; $P=0 \cdot 030)$ among non-drinking subjects. When consuming alcohol, $G G$ subjects had lower plasma Hcy levels compared with $A A+A G$ subjects. Physical activity interacted significantly with $M T R 2756 \mathrm{~A} \rightarrow \mathrm{G}$ in determining plasma Hcy ( $P$ for interaction $=0 \cdot 002)$. Smoking interacted with physical activity for plasma Hcy ( $P$ for interaction $=0 \cdot 023$ )

Conclusions: Smoking and drinking were associated plasma Hcy concentrations. Genetic variants involved in folate metabolism further modify the effects of lifestyle on plasma Hcy.
\end{abstract}

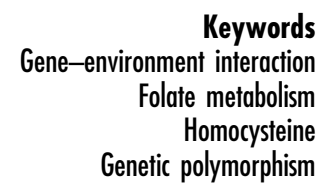

Plasma homocysteine (Hcy) is a thiol-containing amino acid product of methionine metabolism ${ }^{(1)}$. Methionine is converted to $S$-adenosylmethionine via methionine adenosyltransferase ${ }^{(2)}$. $S$-Adenosylhomocysteine, a product of this methyl-transferase reaction, is hydrolysed to Hcy in a reversible reaction via the $S$-adenosylhomocysteine hydrolases. Once synthesized, Hcy can be converted back to methionine in the remethylation pathway via 5-methyltetrahydrofolate reductase (MTHFR) and methionine synthase (MTR) using cofactors such as vitamin $\mathrm{B}_{12}$ and folate ${ }^{(3)}$. Hcy can also be condensed with serine to form cystathionine via vitamin $\mathrm{B}_{6}$-dependent cystathionine $\beta$-synthase in the transsulfuration pathway; subsequently, cystathionine is converted to cysteine, $\alpha$-ketosuccinic acid, taurine and $\mathrm{H}_{2} \mathrm{~S}$ via vitamin $\mathrm{B}_{6}$-dependent cystathionine $\gamma$-lyase ${ }^{(4)}$.
Elevated plasma Hcy concentration is generally considered an independent risk factor for various pathologies such as CVD, endothelial damage, venous thrombosis, atherosclerosis, abnormal collagen cross-linking, oxidative stress, osteoporotic fracture, preterm birth, neural-tube defects, pregnancy complications and several disorders of the central nervous system ${ }^{(5-9)}$, but the causes of high plasma Hcy are not fully understood. The aetiology of hyperhomocysteinaemia (HHcy) is considered to be multi-factorial, including dietary and lifestyle and genetic factors $^{(10)}$. In the USA, two-thirds of cases with HHcy in an elderly population were associated with inadequate plasma/serum concentrations of one or more of the vitamin B group ${ }^{(11)}$. In addition, low intake of $n-3$ PUFA $^{(12-14)}$, smoking $^{(15,16)}$, drinking ${ }^{(17,18)}$ and physical activity ${ }^{(19)}$ were 
also related to elevated plasma Hcy. The genetic causes of HHcy include rare inborn errors of Hcy metabolism, such as variants affecting cystathionine $\beta$-synthase and MTHFR $^{(20-22)}$. Genetic polymorphisms in folate metabolism genes have been reported to be associated with elevated Hcy levels ${ }^{(22,23)}$. Of the genes involved in folate uptake and retention, that encoding folate hydrolase 1 (FOLH1), which hydrolyses dietary folate, has received the most attention with regard to its potential to modulate plasma folate status $^{(22)}$. Studies reported that the $1561 \mathrm{C} \rightarrow \mathrm{T}$ singlenucleotide polymorphism (SNP) in FOLH1 was associated with elevated plasma folate concentrations ${ }^{(24-26)}$. Genetic variation in the proton-coupled folate transporter (PCFI), which was recently identified to absorb folate from the gut $^{(27)}$, may also be associated with plasma folate and Hcy concentrations. We previously also reported that methionine adenosyltransferase variants (MAT1A) affect plasma Hcy levels and $\mathrm{CVD}^{(28)}$.

Although numerous studies have investigated the relationship between lifestyle factors such as smoking, drinking and physical activity, and genetic variants involved in folate metabolism and plasma Hcy levels ${ }^{(16)}$, the results are inconsistent across different populations. Little is known about the combined effects of lifestyle factors and genetic polymorphisms on plasma Hcy in the general population. In the present study, we hypothesized that genetic variants in genes involved in the folate metabolic pathway modulate the effects of smoking, drinking and physical activity on plasma Hcy concentration. The goal of the present study was to examine the interactions between lifestyle factors and selected genetic variants in genes of the folate metabolic pathway in relationship to plasma Hcy in an adult population of Puerto Ricans.

\section{Methods}

\section{Study design and subjects}

The current study was conducted in the ongoing Boston Puerto Rican Health Study (BPRHS) as described previously $^{(29)}$. Briefly, areas of high Hispanic density in the Boston metropolitan area were identified from the year 2000 census, and one Puerto Rican adult from each household with at least one Puerto Rican person between 45 and 75 years of age was randomly selected for participation. Nine hundred and ninety-four participants with complete phenotypes and genotype data were included in the present study. Interviews were conducted in the home. In addition to health-related and anthropometric data, detailed dietary intake data were collected using a questionnaire previously adapted from the National Cancer Institute/Block food frequency form and validated for this population ${ }^{(30)}$. Fasting blood samples were collected the morning following the health interviews in the volunteer's home. Approval for the BPRHS was obtained from the Institutional Review Board of the Tufts Medical Center and Tufts University Health Sciences.

\section{Genetic analysis}

DNA was isolated from blood samples using QIAamp DNA Blood Mini kits according to the manufacturer's instructions (Qiagen, Valencia, CA, USA). Seven SNP of four methionine metabolism genes - MTHFR 1298A $\rightarrow$ C $(\mathrm{rs} 1801131), \quad$ MTHFR $\quad 677 \mathrm{C} \rightarrow \mathrm{T} \quad(\mathrm{rs} 1801133), \quad$ FOLH1 $1561 \mathrm{C} \rightarrow \mathrm{T}(\mathrm{rs} 202712)$, FOLH1 (rs647370), MAT1A 3U150 (rs7087728), MAT1A_i15752 (rs4933327) and PCFT $928 \mathrm{~A} \rightarrow \mathrm{G}$ (rs2239907) - chosen for genotyping are listed in Supplemental Table 1. These variants were genotyped using the TaqMan SNP genotyping system (Applied Biosystems, Foster City, CA, USA) ${ }^{(28)}$. Based on our internal quality control and that estimated independently by external laboratories, the genotyping error rate was $<1 \%$.

\section{Measurement of antbropometric and plasma biochemical parameters}

Anthropometric data including height and weight were measured in duplicate consistent with the technique used by the National Health and Nutrition Examination Survey $^{(31)}$. BMI was calculated as weight in kilograms divided by the square of height in metres. Physical activity was estimated as physical activity score using the Paffenbarger questionnaire of the Harvard Alumni Activity Survey $^{(32)}$. The physical activity score is constructed by weighting time spent in various activities by factors that parallel increasing oxygen consumption rates associated with physical activity intensity ${ }^{(33)}$. Fasting blood samples were collected by venepuncture from all participants. Total plasma Hcy was measured using HPLC with fluorescence detection as previously described ${ }^{(34)}$. Plasma pyridoxal phosphate (PLP) was determined using the radio-enzymatic method of Camp et al. ${ }^{(35)}$. Plasma folate and vitamin $\mathrm{B}_{12}$ were measured using Immulite chemiluminescent kits according to the manufacturer's instructions (Diagnostic Products Corporation/Siemens, Los Angeles, CA, USA).

\section{Dietary assessment}

Dietary intake was assessed using an FFQ that was designed for and validated in this population ${ }^{(30)}$. Dietary data were linked to the Minnesota Nutrient Data System 1999, version 25 (University of Minnesota Nutrition Coordinating Center, Minneapolis, MN, USA) for nutrient analysis. Intakes of fatty acids were expressed as percentages of total energy intake.

\section{Statistical analyses}

The data analyses were performed using the SAS for Windows statistical software package version 9·1 (SAS Institute, Cary, NC, USA). All continuous variables were examined for normal distribution. Men and women were analysed together to ensure adequate statistical power. 
The $\chi^{2}$ test was used to examine whether the genotype frequencies of the selected SNP were in Hardy-Weinberg equilibrium. Correlations between smoking status, plasma parameters and anthropometric measures were performed using ANOVA. Interactions between lifestyle factors and genotypes were tested in a general linear model while adjusting for potential confounders (age, sex, BMI, population admixture, drinking, energy intake, plasma folate, plasma vitamin $\mathrm{B}_{12}$ and plasma PLP, MTHFR $1298 \mathrm{~A} \rightarrow \mathrm{C}$, MTHFR $677 \mathrm{C} \rightarrow \mathrm{T}$ ). The main effect of lifestyles as categorical or binary variables and genotypes as categorical variables were included in the model. All data are expressed as mean and standard deviation. Differences between groups were considered to be statistically significant at $P \leq 0 \cdot 05$.

\section{Population admixture}

For BPRHS participants, population admixture was estimated using principal component analysis ${ }^{(36,37)}$. We estimated population admixture based on a panel of 100 SNP as informative ancestral markers that were genotyped in this population ${ }^{(37)}$. All analyses were adjusted for the estimated population admixture using the first major principal component with linear regression models ${ }^{(37)}$.

\section{Results}

\section{Demographic, antbropometric and biochemical data in Boston Puerto Rican population}

All seven SNP, where minor allele frequencies ranged from 0.05 to $0 \cdot 42$, were in Hardy-Weinberg equilibrium ( $\chi^{2}$ test; Supplemental Table 1). Plasma Hcy, plasma folate and BMI differed significantly between men and women $(P<0 \cdot 01$; Table 1$)$. Plasma Hcy concentration for all subjects ranged from 3.9 to $30.4 \mu \mathrm{mol} / \mathrm{l}$. Men had higher Hcy levels than women $(P<0 \cdot 001$; Table 1). In addition, plasma Hcy was positively correlated with age $(P<0 \cdot 001)$.

\section{Associations between lifestyle and plasma bomocysteine}

Plasma Hcy $(P=0 \cdot 001)$, folate $(P=0 \cdot 011)$ and vitamin $\mathrm{B}_{12}$ $(P=0 \cdot 012)$ were significantly associated with smoking status. Current smokers had higher plasma Hcy (9.64 (SD 4.18) $\mu \mathrm{mol} / \mathrm{l}$ ), but lower plasma folate and PLP compared with non-smokers (Table 2 ). We did not observe significant associations between drinking status and plasma Hcy, folate, PLP and vitamin $\mathrm{B}_{12}$ when drinking status was compared across three groups (current drinkers, former drinkers and non-drinkers) or when current drinkers were compared with non-drinkers and former drinkers combined (Table 2). Physical activity was positively associated with plasma PLP $(P=0 \cdot 001)$ and vitamin $\mathrm{B}_{12}(P=0 \cdot 041)$, but not with plasma Hcy $(P=0 \cdot 068$; Table 2$)$.

\section{Interactions between smoking status and genetic variants on plasma bomocysteine}

Four polymorphisms (MTHFR 677C $\rightarrow$ T, FOLH1 1561C $\rightarrow$ T, FOLH1 rs647370, PCFT 928A $\rightarrow \mathrm{G}$ ) interacted significantly with smoking in determining plasma Hcy while adjusted for age, sex, drinking, BMI, plasma folate, PLP and vitamin $B_{12}$, dietary energy and population admixture $(P=0 \cdot 002$ to $0 \cdot 038$; Table 3$)$. In subjects with the genotype FOLH1 1561TT, plasma Hcy concentrations were significantly different among the three smoking statuses ( $P$ for trend $=0 \cdot 011$ ); subjects who were smokers had the highest plasma Hcy, while subjects who never smoked had the lowest plasma Hcy. However, in subjects with FOLH1 1561CT genotype, there was no significant difference in plasma Hcy levels among the three smoking statuses ( $P$ for trend $=0 \cdot 631$ ). For $P C F T$ 928A $\rightarrow \mathrm{G}$, in $G G$ subjects smoking significantly affected the plasma Hcy

Table 1 Demographic, anthropometric and biochemical characteristics of participants by sex among men and women in the Boston Puerto Rican Health Study

\begin{tabular}{|c|c|c|c|c|}
\hline \multirow[b]{2}{*}{ Characteristic } & \multicolumn{2}{|c|}{ Men ( $n$ 292) } & \multicolumn{2}{|c|}{ Women ( $n$ 702) } \\
\hline & Mean or $n$ & SD or $\%$ & Mean or $n$ & SD or $\%$ \\
\hline Age (years) & $57 \cdot 6$ & $7 \cdot 6$ & $57 \cdot 8$ & $7 \cdot 2$ \\
\hline BMI $\left(\mathrm{kg} / \mathrm{m}^{2}\right)$ & $29 \cdot 7$ & $5 \cdot 1$ & $33 \cdot 0^{*}$ & $6 \cdot 9$ \\
\hline Current smoker, $n(\%)$ & 80 & $31 \cdot 1$ & $126^{\star}$ & $19 \cdot 8$ \\
\hline Current drinker, $n(\%)$ & 132 & $51 \cdot 3$ & $219^{*}$ & $34 \cdot 4$ \\
\hline Physical activity (score) & $32 \cdot 6$ & $5 \cdot 7$ & $31 \cdot 1$ & 3.9 \\
\hline Alcohol (g/d) & $9 \cdot 2$ & $30 \cdot 4$ & $1 \cdot 5^{\star}$ & $6 \cdot 5$ \\
\hline Energy intake $(\mathrm{kJ} / \mathrm{d})$ & 11277 & 5528 & 9099 & 4666 \\
\hline Energy intake (kcal/d) & $2695 \cdot 8$ & $1321 \cdot 3$ & $2174 \cdot 6^{\star}$ & $1115 \cdot 2$ \\
\hline Total fat ( $\%$ of energy) & $31 \cdot 9$ & $5 \cdot 4$ & $30 \cdot 8^{\star}$ & $5 \cdot 2$ \\
\hline Plasma folate $(\mathrm{ng} / \mathrm{ml})$ & $17 \cdot 7$ & $8 \cdot 7$ & $20 \cdot 1^{*}$ & $9 \cdot 4$ \\
\hline Plasma vitamin $\mathrm{B}_{12}(\mathrm{pg} / \mathrm{ml})$ & $526 \cdot 5$ & $276 \cdot 1$ & $549 \cdot 6$ & $284 \cdot 3$ \\
\hline Plasma PLP (nmol/l) & $61 \cdot 4$ & $20 \cdot 3$ & $59 \cdot 2$ & $23 \cdot 3$ \\
\hline Plasma Hcy $(\mu \mathrm{mol} / /)$ & $10 \cdot 7$ & $6 \cdot 2$ & $8 \cdot 8^{\star}$ & $4 \cdot 2$ \\
\hline
\end{tabular}

PLP, pyridoxal phosphate; Hcy, homocysteine.

Data are presented as mean and SD or $n$ and \%

*Significantly different from men $(P<0 \cdot 01)$. 
Table 2 Association between lifestyle and plasma vitamins and metabolites among men and women in the Boston Puerto Rican Health Study

\begin{tabular}{|c|c|c|c|c|c|c|c|c|c|}
\hline \multirow[b]{2}{*}{ Lifestyle factor } & & \multicolumn{2}{|c|}{$\begin{array}{l}\text { Plasma Hcy } \\
(\mu \mathrm{mol} / \mathrm{l})\end{array}$} & \multicolumn{2}{|c|}{$\begin{array}{l}\text { Plasma folate } \\
\text { (ng/ml) }\end{array}$} & \multicolumn{2}{|c|}{$\begin{array}{l}\text { Plasma vitamin } B_{12} \\
(p g / m l)\end{array}$} & \multicolumn{2}{|c|}{$\begin{array}{l}\text { Plasma PLP } \\
(\mathrm{nmol} / \mathrm{l})\end{array}$} \\
\hline & & Mean & SD & Mean & SD & Mean & SD & Mean & SD \\
\hline \multirow[t]{2}{*}{ Smoking status } & Non-smokert (n 753) & $9 \cdot 0$ & 3.5 & $20 \cdot 0$ & $7 \cdot 0$ & $552 \cdot 1$ & $281 \cdot 3$ & $63 \cdot 16$ & $24 \cdot 34$ \\
\hline & $\begin{array}{l}\text { Current smoker ( } n \text { 241) } \\
P \ddagger\end{array}$ & & 0.001 & 0.011 & $9 \cdot 0$ & $512 \cdot 3$ & $241 \cdot 1$ & $\begin{array}{r}49 \cdot 66 \\
0\end{array}$ & 0.068 \\
\hline Drinking status & $\begin{array}{l}\text { Non-drinker§ ( } n \text { 527) } \\
\text { Current drinker (n 349) } \\
P \|\end{array}$ & $\begin{array}{l}9 \cdot 1 \\
9 \cdot 4 \\
0 .\end{array}$ & $\begin{array}{l}3 \cdot 6 \\
4 \cdot 3\end{array}$ & $\begin{array}{l}19 \cdot 5 \\
19 \cdot 0 \\
0\end{array}$ & $\begin{array}{l}9 \cdot 4 \\
8 \cdot 8\end{array}$ & $\begin{array}{l}535 \cdot 4 \\
554 \cdot 8\end{array}$ & $\begin{array}{l}249 \cdot 0 \\
302 \cdot 7\end{array}$ & $\begin{array}{l}56 \cdot 9 \\
64 \cdot 1 \\
0\end{array}$ & $\begin{array}{l}26 \cdot 2 \\
29 \cdot 4 \\
1\end{array}$ \\
\hline Physical activity & $\begin{array}{l}\text { PA score } \leq 30(n \text { 392) } \\
\text { PA score }>30(n 498) \\
P\end{array}$ & $\begin{array}{l}9 \cdot 5 \\
9 \cdot 0 \\
0 .\end{array}$ & $\begin{array}{l}3 \cdot 7 \\
3 \cdot 9\end{array}$ & $\begin{array}{r}18 \cdot 8 \\
19 \cdot 7 \\
0\end{array}$ & $\begin{array}{l}9 \cdot 7 \\
8 \cdot 8\end{array}$ & $\begin{array}{l}520 \cdot 8 \\
559 \cdot 9\end{array}$ & $\begin{array}{l}273 \cdot 7 \\
287 \cdot 5\end{array}$ & $\begin{array}{l}52 \cdot 4 \\
65 \cdot 6 \\
0\end{array}$ & $\begin{array}{l}19 \cdot 2 \\
20 \cdot 6 \\
1\end{array}$ \\
\hline
\end{tabular}

Hcy, homocysteine; PLP, pyridoxal phosphate; PA, physical activity.

tCurrent smokers compared with former and never smokers combined.

$\ddagger$ Adjusted for age, sex, BMI, population admixture, drinking, energy intake, plasma folate, plasma vitamin $\mathrm{B}_{12}$ and plasma PLP, MTHFR $1298 \mathrm{~A} \rightarrow \mathrm{C}$ and MTHFR $677 \mathrm{C} \rightarrow \mathrm{T}$.

SCurrent drinkers compared with former and never drinkers combined.

IIAdjusted for age, sex, BMI, population admixture, smoking, dietary energy, plasma folate, plasma vitamin $\mathrm{B}_{12}$ and plasma PLP, MTHFR $1298 \mathrm{~A} \rightarrow \mathrm{C}$ and MTHFR $677 \mathrm{C} \rightarrow \mathrm{T}$.

TAdjusted for age, sex, BMI, population admixture, smoking, drinking, dietary energy, plasma folate, plasma vitamin $B_{12}$ and plasma PLP, MTHFR $1298 \mathrm{~A} \rightarrow \mathrm{C}$ and $M T H F R$ 677C $\rightarrow$ T.

Table 3 Effect of the interaction between folate metabolic gene polymorphisms and smoking status on plasma homocysteine (Hcy) among men and women in the Boston Puerto Rican Health Study

\begin{tabular}{|c|c|c|c|c|c|c|c|c|c|}
\hline & & \multicolumn{6}{|c|}{ Plasma Hcy $(\mu \mathrm{mol} / \mathrm{l})$} & \multirow[b]{3}{*}{$P$ for trendt } & \multirow{3}{*}{$\begin{array}{c}P \text { for } \\
\text { interaction }\end{array}$} \\
\hline & & \multicolumn{2}{|c|}{ Non-smoker ( $n$ 451) } & \multicolumn{2}{|c|}{ Past smoker ( $n$ 302) } & \multicolumn{2}{|c|}{ Current smoker ( $n$ 241) } & & \\
\hline \multicolumn{2}{|c|}{ Single-nucleotide polymorphism } & Mean & SD & Mean & SD & Mean & SD & & \\
\hline \multirow{2}{*}{ MTHFR $677 \mathrm{C} \rightarrow \mathrm{T}$} & $C C$ & $8 \cdot 7$ & $3 \cdot 7$ & $9 \cdot 2$ & $3 \cdot 7$ & $9 \cdot 6$ & $4 \cdot 5$ & 0.425 & 0.002 \\
\hline & $C T+T T$ & $8 \cdot 9$ & $3 \cdot 7$ & $9 \cdot \overline{5}$ & $3 \cdot 7$ & $9 \cdot 8$ & 3.9 & $0 \cdot 192$ & \\
\hline \multirow{2}{*}{ MTHFR $1298 \mathrm{~A} \rightarrow \mathrm{C}$} & $A A$ & $8 \cdot 7$ & $4 \cdot 0$ & $9 \cdot 5$ & $3 \cdot 7$ & $9 \cdot 6$ & $4 \cdot 3$ & 0.235 & 0.332 \\
\hline & $A C+C C$ & $9 \cdot 1$ & $3 \cdot 7$ & $9 \cdot 3$ & $3 \cdot 7$ & $9 \cdot 7$ & 3.9 & 0.546 & \\
\hline \multirow[t]{2}{*}{ FOLH1 $1561 \mathrm{C} \rightarrow \mathrm{T}$} & $C T$ & $9 \cdot 4$ & $4 \cdot 6$ & $9 \cdot 2$ & $4 \cdot 0$ & $9 \cdot 5$ & 3.9 & 0.631 & 0.038 \\
\hline & $T T$ & $8 \cdot 3$ & $2 \cdot 9$ & $9 \cdot 5$ & $3 \cdot 7$ & $10 \cdot 0$ & $4 \cdot 7$ & 0.011 & \\
\hline \multirow[t]{2}{*}{ FOLH1 (rs647370) } & $G G$ & $8 \cdot 7$ & $3 \cdot 3$ & $9 \cdot 4$ & $3 \cdot 6$ & $9 \cdot 5$ & $3 \cdot 7$ & 0.422 & 0.024 \\
\hline & $A G+A A$ & $9 \cdot 1$ & $4 \cdot 4$ & $9 \cdot 3$ & 3.9 & $10 \cdot 0$ & $4 \cdot 6$ & 0.304 & \\
\hline \multirow[t]{2}{*}{$P C F T$ 928A $\rightarrow \mathrm{G}$} & $G G$ & $8 \cdot 7$ & $3 \cdot 6$ & $9 \cdot 4$ & $3 \cdot 3$ & $9 \cdot 9$ & $4 \cdot 5$ & 0.050 & 0.029 \\
\hline & $A G+A A$ & $9 \cdot 0$ & $4 \cdot 0$ & $9 \cdot 3$ & $3 \cdot 8$ & $9 \cdot 5$ & $4 \cdot 0$ & 0.795 & \\
\hline
\end{tabular}

MTHFR, 5-methyltetrahydrofolate reductase; FOLH1, folate hydrolase 1; PCFT, proton-coupled folate transporter.

†Adjusted for age, sex, BMI, population admixture, drinking, dietary energy, plasma folate, plasma vitamin $\mathrm{B}_{12}$ and plasma pyridoxal phosphate, MTHFR $1298 \mathrm{~A} \rightarrow \mathrm{C}$ and MTHFR $677 \mathrm{C} \rightarrow \mathrm{T}$. After adjusting for $n-3$ PUFA in the model, the results remained the same.

level ( $P$ for trend $=0 \cdot 050$ ), while we did not find any significant effect in subjects with $P C F T$ 928AG or $A A$ ( $P$ for trend $=0 \cdot 795$; Table 3 ).

\section{Interactions between drinking status and PCFT $\mathbf{9 2 8 A} \rightarrow G$ on plasma bomocysteine}

$P C F T$ 928A $\rightarrow \mathrm{G}$ displayed significant interactions with drinking status for plasma Hcy $(P=0 \cdot 002)$ after adjusting for age, sex, smoking, population admixture, dietary energy, dietary total fat, plasma folate, plasma vitamin $\mathrm{B}_{12}$ and plasma PLP. Further analysis showed that $G G$ homozygotes had significantly higher plasma Hcy concentrations compared with homozygous $(A A)$ and heterozygous $(A G)$ subjects combined $(P=0 \cdot 030)$ among non-drinkers. Among current drinkers, $G G$ homozygotes had lower plasma Hcy concentrations when compared with homozygous $(A A)$ and heterozygous ( $A G$ ) subjects (Fig. 1).

\section{Influence on plasma bomocysteine of the interaction between physical activity and the MTR 2756A $\rightarrow$ G/smoking association}

MTR $2756 \mathrm{~A} \rightarrow \mathrm{G}$ significantly interacted with physical activity in influencing plasma Hcy level $(P=0 \cdot 002)$. When the physical activity score was low $(<40)$, there was no significant difference between $M T R 2756 A A$ and minor allele $(A G+G G)$ carriers. Interestingly, minor allele carriers had significantly higher plasma Hcy than homozygous $(A A)$ subjects $(P=0.034)$ when the physical activity score was $\geq 40$ (Fig. 2 ). Smoking interacted significantly with physical activity to influence plasma Hcy level $(P=0 \cdot 023$; Fig. 3$)$. When the physical activity score was low $(<40)$, there was no significant difference between Hcy concentrations in smokers and nonsmokers. However, when physical activity score was high $(\geq 40)$, we observed that minor allele carriers $(A G+G G)$ 


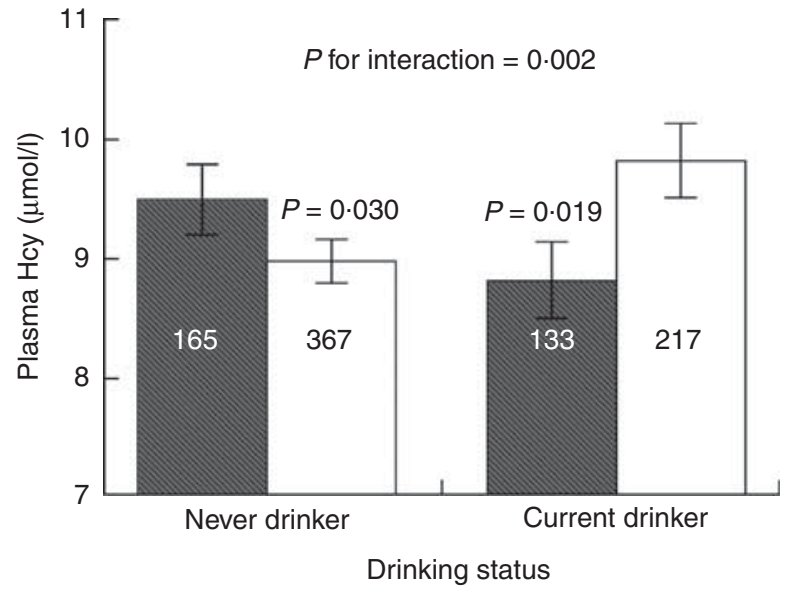

Fig. 1 Effect of the interaction between drinking status and PCFT (proton-coupled folate transporter) genotype (PCFT 928G $\rightarrow \mathrm{A}$ : $\square, G G ; \square, A G+A A$ ) on plasma homocysteine (Hcy) among men and women in the Boston Puerto Rican Health Study. Values are means with their standard deviations represented by vertical bars for the number of subjects given. Former drinkers and never drinkers were combined for comparison of plasma Hcy with current drinkers. Results were adjusted for age, sex, BMI, population admixture, smoking, energy intake, plasma folate, plasma vitamin $\mathrm{B}_{12}$, plasma pyridoxal phosphate, MTHFR $1298 \mathrm{~A} \rightarrow \mathrm{C}$ and MTHFR $677 \mathrm{C} \rightarrow \mathrm{T}$

had significantly higher plasma Hcy than major allele homozygotes ( $A A ; P=0 \cdot 012$ ).

\section{Discussion}

In the present study conducted in the BPRHS, we demonstrated that lifestyle factors such as smoking were associated with elevated plasma Hcy concentration but that drinking and physical activity were not directly associated with plasma Hcy. Moreover, we observed that smoking, drinking and physical activity each interacted with genetic polymorphisms in genes involved in the methionine metabolic pathway in determining plasma Hcy levels.

Elevated plasma Hcy plays an important role in the pathology of $\mathrm{CVD}^{(3)}$. HHcy is a complex, multi-factorial condition and its environmental and genetic contributors continue to be identified and refined. However, these factors often have been evaluated mainly independently of one another. In the present study, we examined previously unreported interactions between lifestyle and genetic variants in modulating plasma Hcy levels.

Plasma Hcy concentrations were significantly higher in men than in women, which is consistent with previous studies $^{(16,38)}$. This sex difference can be explained in part by folate intake, as the plasma folate concentrations in women were significantly higher than in men in this population. Other factors such as sex hormones may also contribute to gender-related differences in Hcy. Furthermore, plasma Hcy is higher in postmenopausal women

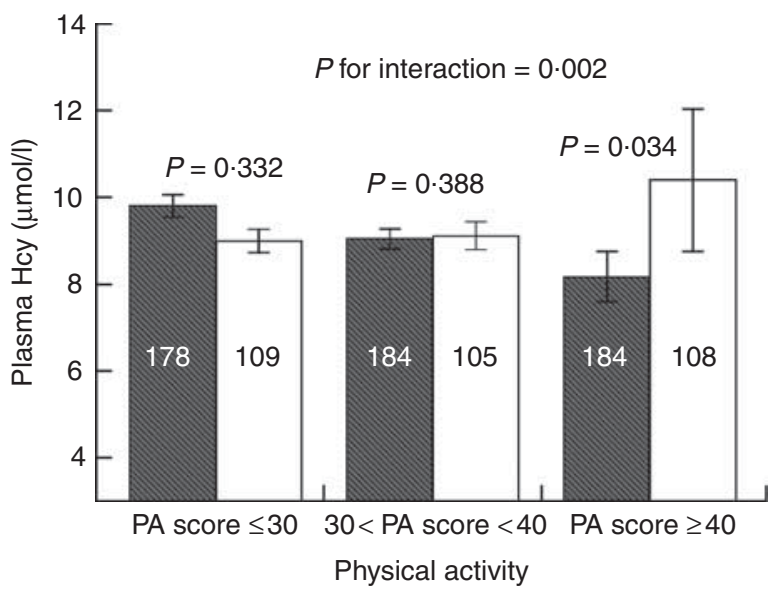

Fig. 2 Effect of the interaction between physical activity and MTR (methionine synthase) genotype (MTR 2756A $\rightarrow$ G: $\square$, $A A ; \square, A G+G G)$ on plasma homocysteine (Hcy) among men and women in the Boston Puerto Rican Health Study. Values are means with their standard deviations represented by vertical bars for the number of subjects given. Minor allele carriers $(A G+G G)$ were compared with homozygous subjects $(A A)$ under three categories of physical activity score (PA score) for plasma Hcy. Results were adjusted for age, sex, BMI, population admixture, smoking, drinking, energy intake, plasma folate, plasma vitamin $B_{12}$, plasma pyridoxal phosphate, MTHFR $1298 \mathrm{~A} \rightarrow \mathrm{C}$ and MTHFR $677 \mathrm{C} \rightarrow \mathrm{T}$

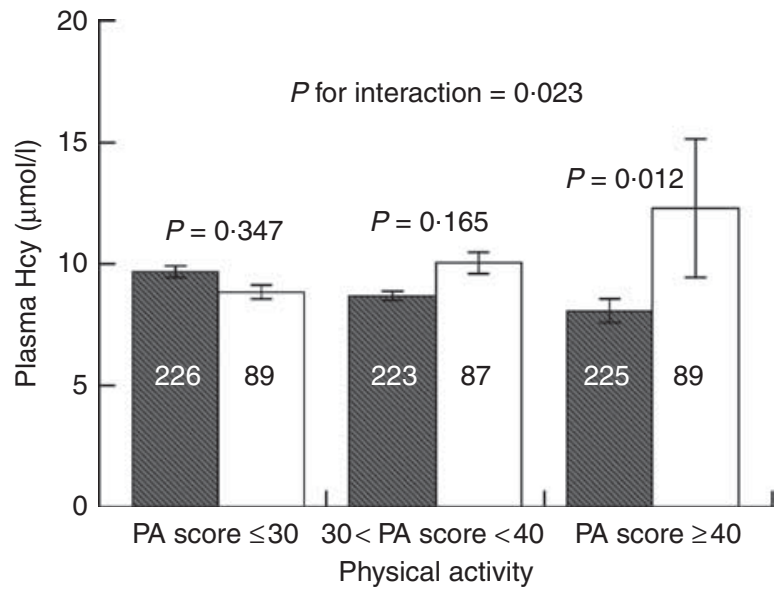

Fig. 3 Effect of the interaction between physical activity and smoking status ( $\square$, non-smokers; $\square$, smokers) on plasma homocysteine (Hcy) level among men and women in the Boston Puerto Rican Health Study. Values are means with their standard deviations represented by vertical bars for the number of subjects given. Non-smokers were compared with smokers under three categories of physical activity score (PA score) for plasma Hcy. Results were adjusted for age, sex, $\mathrm{BMI}$, population admixture, drinking, energy intake, plasma folate, plasma vitamin $B_{12}$, plasma pyridoxal phosphate, MTHFR $1298 \mathrm{~A} \rightarrow \mathrm{C}$ and MTHFR $677 \mathrm{C} \rightarrow \mathrm{T}$

compared with premenopausal women and is reduced in response to oestrogen replacement therapy ${ }^{(34,35)}$. All of these factors may contribute to the higher plasma Hcy concentrations observed in men compared to women. 
In accordance with other studies in large populations $^{(14,36)}$ and a randomized control trial ${ }^{(39)}$, we also observed that cigarette smoking was positively associated with plasma Hcy concentrations, while smoking was negatively associated with plasma folate and PLP. The mechanism for this relationship is not fully understood, but one possibility is smoking might reduce the availability of folate for the remethylation of Hcy to methionine ${ }^{(40)}$. Additional proposed mechanisms are smoking, which induces local effects in cells exposed to cigarette smoke, may influence the Hcy concentration by changing plasma thiol redox status, or could inhibit enzymes involved in the metabolism of $\mathrm{Hcy}^{(40)}$.

In the present study, we observed the same interaction between smoking status and MTHFR $677 \mathrm{C} \rightarrow \mathrm{T}$ as reported previously $^{(14)}$. In addition, we identified previously unreported interactions between smoking and genetic polymorphisms (FOLH1 1561C $\rightarrow$ T, FOLH1 rs647370, PCFT 928A $\rightarrow \mathrm{G}$ ) for plasma Hcy. The suboptimal plasma folate status among smokers observed in our study might explain this interaction, because plasma folate has been shown to interact with MTHFR $677 \mathrm{C} \rightarrow \mathrm{T}^{(15)}$. Another study which reported that smoking interacted with dietary folate further supports this relationship ${ }^{(41)}$.

Moderate alcohol consumption in social drinkers increased plasma Hcy levels ${ }^{(18)}$. MTHFR 677T, MTRR 66A, GCPII $1561 \mathrm{~T}$ and alcohol intake were also associated with higher plasma Hcy among South Indians ${ }^{(16)}$. However, we did not confirm these results in the present study. One possible explanation for this discrepancy is genetic variants involved in folate metabolism may modify the effect of drinking on plasma Hcy and folate levels. Therefore, to evaluate our hypothesis, we examined the interactions of drinking and critical genes involved in folate metabolism on plasma Hcy. Interestingly, we observed that PCFT 928A $\rightarrow \mathrm{G}$ interacts significantly with drinking status for plasma Hcy, which suggests that the effect of drinking on plasma Hcy depended on PCFT genotype, and may account for the inconsistencies with previous studies. Studies also suggested that higher plasma Hcy concentrations in consumers of large quantities of alcohol may be related to trapping of 5-methyltetrahydrofolate by alcohol and impaired remethylation associated with genetic polymorphisms ${ }^{(17)}$ or to ethanol-induced B vitamin depletion ${ }^{(40)}$ and interference of alcohol with intestinal absorption of folate ${ }^{(42)}$. Therefore, these results suggest that the effect of drinking on plasma Hcy depends on folate metabolism-related gene polymorphisms.

Despite the inconsistent results of effects of physical activity on plasma Hcy levels, several studies have reported beneficial effects on Hcy in response to exercise. Plasma Hcy level is inversely related to level of physical activity, especially in older subjects ${ }^{(38)}$. In contrast, other studies reported that physical exercise does not reduce plasma Hcy levels or may even be associated with higher concentrations $^{(43)}$. In the present study we also observed that physical activity was not associated with plasma Hcy.
Therefore, questions about the nature of the relationship between Hcy and physical activity remain unanswered ${ }^{(43)}$. However, these inconsistencies reported in previous studies can be explained by our present results which identified a previously unreported interaction between MTR $2756 \mathrm{~A} \rightarrow \mathrm{G}$ and physical activity on Hcy. This result suggests that not all subjects in a population have the same response to physical activity, as the effect of physical activity on plasma Hcy depends on MTR genotype. Additionally, it has been shown that the thermolabile variant of $M T H F R \quad 677 \mathrm{C} \rightarrow \mathrm{T}$ (rs1801133, a regulating enzyme in Hcy metabolism) modified the relationship between several lifestyle factors and $\mathrm{Hcy}^{(44)}$. Thus, the effect of the changes in lifestyle-related factors on Hcy may depend on MTHFR C677T genotype ${ }^{(45)}$. Better understanding of the complex relationships between lifestyle and Hcy will require additional studies focusing on the interaction between physical activity and genetic polymorphisms involved in folate metabolic pathways. The biological mechanism through which physical activity lowers plasma Hcy levels remains to be determined.

In summary, the present study reinforces earlier evidence demonstrating that lifestyle factors are important modifiers of plasma Hcy concentration. In addition, our work extends this knowledge to demonstrate that genetic polymorphisms in genes whose proteins function in the methionine metabolic pathway further modulate the effects of lifestyle factors which influence plasma Hcy. It is essential to determine whether changes in these lifestyle factors reinforce effects in the context of interaction with genetic variation on Hcy metabolism and to clarify the potential mechanism(s) by which this can take place.

\section{Acknowledgements}

The current work was supported by the China Scholarship Council; the National Institutes of Health/National Institute on Aging (grant number 5P01AG023394-02); the National Institutes of Health/National Heart, Lung, and Blood Institute (grant numbers HL54776 and HL078885); contracts 53-K06-5-10 and 58-1950-9-001 from the US Department of Agriculture Research Service; and the National Natural Science Foundation of China (grant number 30972464). The authors report no conflict of interest. T.H. and Y.-C.L. carried out the studies, analysed data and drafted the manuscript; C.-Q.L., L.D.P., Y.-C.L., C.E.S., J.S. and J.W.C. participated in manuscript preparation; and C.-Q.L., K.L.T., D.L. and J.M.O. participated in the project design. All authors read and approved the final manuscript.

\section{References}

1. McCully KS (1969) Vascular pathology of homocysteinemia: implications for the pathogenesis of arteriosclerosis. Am J Pathol 56, 111-128. 
2. Kaul S, Zadeh AA \& Shah PK (2006) Homocysteine hypothesis for atherothrombotic cardiovascular disease: not validated. J Am Coll Cardiol 48, 914-923.

3. Huang T, Yuan G, Zhang Z et al. (2008) Cardiovascular pathogenesis in hyperhomocysteinemia. Asia Pac J Clin Nutr 17, 8-16.

4. Selhub J (1999) Homocysteine metabolism. Annu Rev Nutr 19, 217-246.

5. Kluijtmans LA, Young IS, Boreham CA et al. (2003) Genetic and nutritional factors contributing to hyperhomocysteinemia in young adults. Blood 101, 2483-2488.

6. Arnesen E, Refsum H, Bonaa KH et al. (1995) Serum total homocysteine and coronary heart disease. Int J Epidemiol 24, 704-709.

7. Stampfer MJ, Malinow MR, Willett WC et al. (1992) A prospective study of plasma homocyst(e)ine and risk of myocardial infarction in US physicians. JAMA 268, 877-881.

8. Di Santolo M, Banfi G, Stel G et al. (2009) Association of recreational physical activity with homocysteine, folate and lipid markers in young women. Eur J Appl Physiol 105, 111-118.

9. McCully KS \& Wilson RB (1975) Homocysteine theory of arteriosclerosis. Atherosclerosis 22, 215-227.

10. Refsum H, Ueland PM, Nygard O et al. (1998) Homocysteine and cardiovascular disease. Annu Rev Med 49, 31-62.

11. Selhub J, Jacques PF, Rosenberg IH et al. (1999) Serum total homocysteine concentrations in the third National Health and Nutrition Examination Survey (1991-1994): population reference ranges and contribution of vitamin status to high serum concentrations. Ann Intern Med 131, 331-339.

12. Huang T, Wahlqvist ML \& Li D (2010) Docosahexaenoic acid decreases plasma homocysteine via regulating enzyme activity and mRNA expression involved in methionine metabolism. Nutrition 26, 112-119.

13. Li D, Mann NJ \& Sinclair AJ (2006) A significant inverse relationship between concentrations of plasma homocysteine and phospholipid docosahexaenoic acid in healthy male subjects. Lipids $\mathbf{4 1}, 85-89$.

14. Huang T, Tucker K, Lee YC et al. (2010) MAT1A variants modulate the effect of dietary fatty acids on plasma homocysteine concentrations. Nutr Metab Cardiovasc Dis (Epublication ahead of print version).

15. Husemoen LL, Thomsen TF, Fenger M et al. (2004) Effect of lifestyle factors on plasma total homocysteine concentrations in relation to MTHFR(C677T) genotype. Inter99 (7). Eur J Clin Nutr 58, 1142-1150.

16. Saw SM, Yuan JM, Ong CN et al. (2001) Genetic, dietary, and other lifestyle determinants of plasma homocysteine concentrations in middle-aged and older Chinese men and women in Singapore. Am J Clin Nutr 73, 232-239.

17. Vinukonda G, Shaik Mohammad N, Md Nurul Jain J et al (2009) Genetic and environmental influences on total plasma homocysteine and coronary artery disease (CAD) risk among South Indians. Clin Chim Acta 405, 127-131.

18. Bleich S, Bleich K, Kropp S et al. (2001) Moderate alcohol consumption in social drinkers raises plasma homocysteine levels: a contradiction to the 'French paradox'? Alcobol Alcohol 36, 189-192.

19. Chrysohoou C, Panagiotakos DB, Pitsavos C et al. (2004) The associations between smoking, physical activity, dietary habits and plasma homocysteine levels in cardiovascular disease-free people: the 'ATTICA' study. Vasc $\operatorname{Med}$ 9, 117-123.

20. Blom HJ (1998) Determinants of plasma homocysteine. Am J Clin Nutr 67, 188-189.

21. Frosst P, Blom HJ, Milos R et al. (1995) A candidate genetic risk factor for vascular disease: a common mutation in methylenetetrahydrofolate reductase. Nat Genet 10, 111-113.

22. DeVos L, Chanson A, Liu Z et al. (2008) Associations between single nucleotide polymorphisms in folate uptake and metabolizing genes with blood folate, homocysteine, and DNA uracil concentrations. Am J Clin Nutr 88, 1149-1158.

23. Devlin AM, Clarke R, Birks J et al. (2006) Interactions among polymorphisms in folate-metabolizing genes and serum total homocysteine concentrations in a healthy elderly population. Am J Clin Nutr 83, 708-713.

24. Vargas-Martinez C, Ordovas JM, Wilson PW et al. (2002) The glutamate carboxypeptidase gene II $(\mathrm{C}>\mathrm{T})$ polymorphism does not affect folate status in the Framingham Offspring cohort. J Nutr 132, 1176-1179.

25. Lievers KJ, Kluijtmans LA, Boers GH et al. (2002) Influence of a glutamate carboxypeptidase II (GCPII) polymorphism $(1561 \mathrm{C} \rightarrow \mathrm{T})$ on plasma homocysteine, folate and vitamin $\mathrm{B}(12)$ levels and its relationship to cardiovascular disease risk. Atherosclerosis 164, 269-273.

26. Winkelmayer WC, Eberle C, Sunder-Plassmann G et al. (2003) Effects of the glutamate carboxypeptidase II (GCP2 $1561 \mathrm{C}>\mathrm{T}$ ) and reduced folate carrier (RFC1 80G $>$ A) allelic variants on folate and total homocysteine levels in kidney transplant patients. Kidney Int 63, 2280-2285.

27. Qiu A, Jansen M, Sakaris A et al. (2006) Identification of an intestinal folate transporter and the molecular basis for hereditary folate malabsorption. Cell 127, 917-928.

28. Lai CQ, Parnell LD, Troen AM et al. (2010) MAT1A variants are associated with hypertension, stroke, and markers of DNA damage and are modulated by plasma vitamin B-6 and folate. Am J Clin Nutr 91, 1377-1386.

29. Tucker KL, Mattei J, Noel SE et al. (2010) The Boston Puerto Rican Health Study, a longitudinal cohort study on health disparities in Puerto Rican adults: challenges and opportunities. BMC Public Health 10, 107.

30. Tucker KL, Bianchi LA, Maras J et al. (1998) Adaptation of a food frequency questionnaire to assess diets of Puerto Rican and non-Hispanic adults. Am J Epidemiol 148, 507-518.

31. Smith CE, Tucker KL, Yiannakouris N et al. (2008) Perilipin polymorphism interacts with dietary carbohydrates to modulate anthropometric traits in Hispanics of Caribbean origin. J Nutr 138, 1852-1858.

32. Lee IM \& Paffenbarger RS (1998) Physical activity and stroke incidence - The Harvard Alumni Health Study. Stroke 29, 2049-2054.

33. Lai CQ, Tucker KL, Parnell LD et al. (2008) PPARGC1A variation associated with DNA damage, diabetes, and cardiovascular diseases: the Boston Puerto Rican Health Study. Diabetes 57, 809-816.

34. Araki A \& Sako Y (1987) Determination of free and total homocysteine in human plasma by high-performance liquid chromatography with fluorescence detection. J Chromatogr 422, 43-52.

35. Camp VM, Chipponi J \& Faraj BA (1983) Radioenzymatic assay for direct measurement of plasma pyridoxal $5^{\prime}$ phosphate. Clin Chem 29, 642-644.

36. Price AL, Patterson NJ, Plenge RM et al. (2006) Principal components analysis corrects for stratification in genomewide association studies. Nat Genet 38, 904-909.

37. Lai CQ, Tucker KL, Choudhry S et al. (2009) Population admixture associated with disease prevalence in the Boston Puerto Rican health study. Hum Genet 125, 199-209.

38. Nygard O, Vollset SE, Refsum H et al. (1995) Total plasma homocysteine and cardiovascular risk profile. The Hordaland Homocysteine Study. JAMA 274, 1526-1533.

39. Stein JH, Bushara M, Bushara K et al. (2002) Smoking cessation, but not smoking reduction, reduces plasma homocysteine levels. Clin Cardiol 25, 23-26.

40. De Bree A, Verschuren WM, Kromhout D et al. (2002) Homocysteine determinants and the evidence to what extent homocysteine determines the risk of coronary heart disease. Pharmacol Rev 54, 599-618. 
41. de Bree A, Verschuren WM, Blom HJ et al. (2001) Association between B vitamin intake and plasma homocysteine concentration in the general Dutch population aged 20-65 y. Am J Clin Nutr 73, 1027-1033.

42. Halsted CH, Villanueva JA, Devlin AM et al. (2002) Metabolic interactions of alcohol and folate. J Nutr 132, 8 Suppl., 2367S-2372S.

43. Sotgia S, Carru C, Caria MA et al. (2007) Acute variations in homocysteine levels are related to creatine changes induced by physical activity. Clin Nutr 26, 444-449.
44. Jacques PF, Kalmbach R, Bagley PJ et al. (2002) The relationship between riboflavin and plasma total homocysteine in the Framingham Offspring cohort is influenced by folate status and the C677T transition in the methylenetetrahydrofolate reductase gene. J Nutr 132, 283-288.

45. Husemoen LL, Linneberg A, Fenger M et al. (2009) Changes in lifestyle, biological risk factors and total homocysteine in relation to MTHFR C677T genotype: a 5-year follow-up study. Eur J Clin Nutr 63, 1233-1240. 